\title{
ON THE FOURIER TRANSFORM OF THE INDICATOR FUNCTION OF A PLANAR SET $\left({ }^{1}\right)$
}

\author{
BY \\ BURTON RANDOL
}

Suppose $C$ is a compact subset of the plane having a piecewise smooth boundary $\partial C$. Let $F(r, \theta)$ be the Fourier transform, in polar coordinates, of the indicator function of the set $C$, where by the indicator function of $C$, we mean the function whose value on $C$ is 1 , and whose value on the complement of $C$ is 0 . In $\S 1$ of this paper, we shall describe some relationships between geometric properties of $C$, and the asymptotic behavior of $F(r, \theta)$ as $r \rightarrow \infty$. In $\S 2$, we shall give applications of the results of $\S 1$ to some questions in the geometry of numbers.

1. If $\partial C$ is sufficiently smooth, and has everywhere positive Gaussian curvature, it is known that the function $\Phi(\theta)=\sup _{r} r^{3 / 2}|F(r, \theta)|$ is bounded on $S^{1}$ (cf. [1]). If $\partial C$ has points of zero curvature, this need no longer be true (cf. [3]). The following, however, remains true:

THeOREM 1. If $\partial C$ is of class $C^{n+3}$, for some integer $n \geqq 1$, and if the Gaussian curvature of $\partial C$ is nonzero at all points of $\partial C$, with the possible exception of a finite set, at each point of which the tangent line has contact of order $\leqq n$, then $\Phi(\theta)$ is bounded on $S^{1}$, if $n=1$, and of class $L^{p}$ on $S^{1}$, for any $p<2 n /(n-1)$, if $n>1$.

Moreover, $\Phi(\theta)$ is always bounded, except in neighborhoods of those points of $S^{1}$ which, regarded as vectors, correspond to exterior or interior normals to $\partial C$ at points of zero curvature. In a neighborhood of such a point $\theta_{0}, \Phi(\theta)$ is bounded by a multiple of [dist $\left.\left(\theta, \theta_{0}\right)\right]^{-\left(n_{1}-1\right) / 2 n_{1}}$, where dist $\left(\theta, \theta_{0}\right)$ is the length of the smaller arc of $S^{1}$ connecting $\theta$ and $\theta_{0}$, and $n_{j}$ is the largest order of contact which can occur between $\partial C$ and its tangent line, at those points of $\partial C$ at which the exterior normal is either $\theta_{0}$ or $-\theta_{0}$.

REMARK. Theorem 1 has analogues in higher dimensions. I shall show in another paper, by different methods, that if $C$ is a compact convex subset of $R^{n}$, whose boundary is analytic, and if $F(r, \theta)$ is the Fourier transform, in polar coordinates, of the indicator function of the set $C$, then $\sup _{r} r^{(n+1) / 2}|F(r, \theta)|$ is of class $L^{p}$ on $S^{n-1}$, for some $p>2$.

If $C$ is a polygon, the estimates are of a quite different character.

THEOREM 2. Suppose $C$ is a polygon. Then

1. For any $\varepsilon>0$, $\sup _{r} r^{2-\varepsilon}|F(r, \theta)|$ is of class $L^{p}$ on $S^{1}$, for any $p<1 /(1-\varepsilon)$.

2. For any $\varepsilon>0, \sup _{r} r^{2}(\log r)^{-(1+\varepsilon)}|F(r, \theta)|$ is of class $L^{1}$ on $S^{1}$.

Received by the editors August 2, 1968.

$\left.{ }^{1}\right)$ This research was supported by a grant from the Army Research Office, Durham. 
In order to prove Theorems 1 and 2, we require two lemmas.

LEMMA 1. Suppose $b>0$, and suppose $f(x)$ is of the form $h(x) x^{k}+c$ on $[0, b]$, where $h(x)$ is $C^{2}$ and nonvanishing on $[0, b], c$ is a constant, and $k$ is an integer $\geqq 2$. Suppose, moreover, that $b$ is small enough so that $\left|f^{\prime}(x)\right| \leqq c_{1} x^{k-1}$, and $\left|f^{\prime \prime}(x)\right| \geqq c_{2} x^{k-2}$, for some positive constants $c_{1}$ and $c_{2}$. Denote by $S$ the arc traced out in the $\left(x_{1}, x_{2}\right)$-plane by the point $\left(x_{1}, f\left(x_{1}\right)\right)$, as $x_{1}$ runs from 0 to $b$, and suppose $g$ is a function of class $C^{1}$ in a neighborhood of $S$. Define

$$
H(Y)=\int_{S} g(X) \exp (2 \pi i(X, Y)) d S_{X},
$$

where $X=\left(x_{1}, x_{2}\right), Y=\left(y_{1}, y_{2}\right)$, and $d S_{X}=$ the arc length element on $S$. Let $A(Y)$ be the smallest nonnegative angle which the vector $Y \neq 0$ makes with the $x_{2}$-axis. Then there exists $M>0$ such that $|H(Y)| \leqq M|Y|^{-1 / 2}(A(Y))^{-(k-2) /(2 k-2)}$, where $|Y|=\left(y_{1}^{2}+y_{2}^{2}\right)^{1 / 2}$. Moreover, in terms of $g$, bounds for $M$ depend only on bounds for $g$ and its first derivatives on $S$.

Proof. We shall assume that $h(x)<0$ on $[0, b]$, since the proof in the remaining case is essentially the same. Now $S$ is concave downward with respect to the $x_{1}$-axis, and its curvature is never zero, except at the point $(0, f(0))$, and then only if $k>2$. Moreover, the angle $\alpha\left(x_{1}\right)$, which the upward normal to $S$ makes with the positive $x_{2}$-axis, is a decreasing function of $x_{1}$ on $[0, b]$, with $\alpha(b)<\pi / 2$. We shall make the temporary assumption that $Y$ lies in the first quadrant, and that $A(Y) \leqq \alpha(b)$.

For a given $Y=|Y|\left(y_{1}^{*}, y_{2}^{*}\right)$, let $\left(p_{1}, p_{2}\right)$ be the point on $S$ at which the upward normal to $S$ is parallel to $\left(y_{1}^{*}, y_{2}^{*}\right)$. Now the equation for $S$ is $x_{2}-f\left(x_{1}\right)=0$ $(x \in[0, b])$, and taking the gradient of the left side, we find that the vector $\left(-f^{\prime}\left(p_{1}\right), 1\right)$ is parallel to $\left(y_{1}^{*}, y_{2}^{*}\right)$. I.e., $y_{1}^{*} / y_{2}^{*}=-f^{\prime}\left(p_{1}\right)$, and this clearly implies that $\left|f^{\prime}\left(p_{1}\right)\right| \geqq c_{3} A(Y)$, for some $c_{3}>0$ which does not depend on $Y$, and by our hypotheses, this in turn implies that $p_{1} \geqq c_{4}(A(Y))^{1 /(k-1)}$, for some $c_{4}>0$ which does not depend on $Y$.

Now

$$
H(Y)=\int_{0}^{b} g\left(x_{1}, f\left(x_{1}\right)\right) m\left(x_{1}\right) \exp \left(2 \pi i|Y| E\left(x_{1}\right)\right) d x_{1},
$$

where $E\left(x_{1}\right)=x_{1} y_{1}^{*}+f\left(x_{1}\right) y_{2}^{*}$, and $m\left(x_{1}\right)$ represents the distortion of measure which arises from the projection of $S$ onto the $x_{1}$-axis. For convenience of notation, we shall define $G\left(x_{1}\right)=g\left(x_{1}, f\left(x_{1}\right)\right)$.

The integral on the right side of (1) can be split into two parts

$$
\int_{0}^{b}=\int_{0}^{p_{1}}+\int_{p_{1}}^{b} .
$$

We shall investigate the first integral on the right side of (2). The same technique yields an identical estimate for the second integral. To obtain the desired estimate for the first integral, we need some information about $E\left(x_{1}\right)$. To begin with, note 
that it is clear from the geometrical meaning of $E\left(x_{1}\right)$ that $E^{\prime}\left(p_{1}\right)=0$, and that for $0 \leqq x_{1} \leqq p_{1}, E^{\prime}\left(x_{1}\right) \geqq 0$, and $E^{\prime \prime}\left(x_{1}\right) \leqq 0$. (Recall that $S$ is concave downwards, and that $E\left(x_{1}\right)$ is simply the component of the vector $\left(x_{1}, f\left(x_{1}\right)\right)$ in the direction of the upward normal to $S$ at $\left(p_{1}, p_{2}\right)$.) In what follows, we shall assume that $x_{1} \leqq p_{1}$.

Now

$$
E^{\prime}\left(x_{1}\right)=\int_{x_{1}}^{p_{1}}\left|E^{\prime \prime}(t)\right| d t=\int_{x_{1}}^{x_{1}+\lambda / 3}+\int_{x_{1}+\lambda / 3}^{x_{1}+2 \lambda / 3}+\int_{x_{1}+2 \lambda / 3}^{p_{1}},
$$

where $\lambda=p_{1}-x_{1}$.

But it follows from our hypotheses, that $\left|E^{\prime \prime}(t)\right| \geqq c_{5} t^{k-2}$, for some $c_{5}>0$, which does not depend on $Y$, and throughout at least one of the intervals of integration, $t^{k-2}$ must be at least of the order of $p_{1}^{k-2}$, so (3) implies that $E^{\prime}\left(x_{1}\right) \geqq c_{6}\left(p_{1}-x_{1}\right) p_{1}^{k-2}$, for some $c_{6}>0$, and hence, $E^{\prime}\left(x_{1}\right) \geqq c_{7}\left(p_{1}-x_{1}\right)(A(Y))^{(k-2) /(k-1)}$, for some $c_{7}>0$, where the constants do not depend on $Y$.

We now return to the estimation of the first integral on the right side of (2). Set $\delta=|Y|^{-1 / 2}(A(Y))^{-(k-2) /(2 k-2)}$. Then

$$
\int_{0}^{p_{1}}=\int_{0}^{p_{1}-\delta}+\int_{p_{1}-\delta}^{p_{1}}=\int_{0}^{p_{1}-\delta}+O\left(|Y|^{-1 / 2}(A(Y))^{-(k-2) /(2 k-2)}\right) .
$$

Now

$$
\begin{aligned}
\int_{0}^{p_{1}-\delta} G\left(x_{1}\right) & m\left(x_{1}\right) \exp \left(2 \pi i|Y| E\left(x_{1}\right)\right) d x_{1} \\
= & (2 \pi i|Y|)^{-1} \int_{0}^{p_{1}-\delta}\left(E^{\prime}\left(x_{1}\right)\right)^{-1} G\left(x_{1}\right) m\left(x_{1}\right) d\left(\exp \left(2 \pi i|Y| E\left(x_{1}\right)\right)\right)
\end{aligned}
$$

and if we note that $\left(E^{\prime}\left(x_{1}\right)\right)^{-1}$ is increasing, and apply the second mean value theorem to the real and imaginary parts of the integral on the right, we immediately find that this integral is $O\left(|Y|^{-1 / 2}(A(Y))^{-(k-2) /(2 k-2)}\right)$, which proves the lemma if $Y$ is in the first quadrant, and $A(Y) \leqq \alpha(b)$. If $Y$ is in the first quadrant, and $A(Y)>\alpha(b)$, it is a simple matter to show that $E^{\prime}\left(x_{1}\right) \geqq c_{8}\left(b-x_{1}\right)$, for some $c_{8}>0$ which does not depend on $Y$, and the proof then proceeds as before. If $Y$ is in the upper half-plane, but not in the first quadrant, it is evident that the function $\left|E^{\prime}\left(x_{1}\right)\right|$ is larger than it would be if $Y$ were reflected in the $x_{2}$-axis, so the result follows from the case we have already discussed. Finally, the result for $Y$ in the lower half-plane, i.e., for $y_{2} \leqq 0$, clearly follows from the result for $Y$ in the upper half-plane.

Lemma 2. Suppose $S$ is a straight line segment in the $\left(x_{1}, x_{2}\right)$-plane. Define

$$
H(Y)=\int_{S} \exp (2 \pi i(X, Y)) d S_{X}
$$

where, as before, $X=\left(x_{1}, x_{2}\right), Y=\left(y_{1}, y_{2}\right)$, and $d S_{X}=$ the arc length element on $S$. For $Y \neq 0$, let $A(Y)$ be the smallest nonnegative angle which $Y$ makes with the line perpendicular to $S$. Suppose $g(t)$ is a positive function, defined for $t \geqq t_{0}$, and such that 
both $g(t)$ and $t / g(t)$ are nondecreasing over $\left[t_{0}, \infty\right)$. Then there exists $M>0$, such that for $|Y|, 1 / 4 A(Y) \geqq t_{0}$,

$$
|H(Y)| \leqq M g(|Y|)[|Y| A(Y) g(1 / 4 A(Y))]^{-1} .
$$

Proof. It suffices to prove the result for the case in which $A(Y)$ is near zero. Moreover, if we assume, as we may, that $S$ is the segment from -1 to 1 on the $x_{1}$-axis, and that $Y$ lies in the first quadrant, then $H(Y)=\left(\pi y_{1}\right)^{-1} \sin 2 \pi y_{1}$, and $y_{1}=|Y| \sin A(Y)$. I.e., $H(Y)=(\pi|Y| \sin A(Y))^{-1} \sin (2 \pi|Y| \sin A(Y))$, and for $|Y| \geqq t_{0}$,

$$
|Y|(g(|Y|))^{-1} H(Y)=(\pi g(|Y|) \sin A(Y))^{-1} \sin (2 \pi|Y| \sin A(Y)) .
$$

For fixed $A(Y) \neq 0$, consider the supremum, for $|Y| \geqq t_{0}$, of $|Y|(g(|Y|))^{-1}|H(Y)|$. Since $g(t)$ is nondecreasing, it is clear that if $A(Y)$ is small, the supremum may be taken over the interval $t_{0} \leqq|Y| \leqq(4 \sin A(Y))^{-1}$. Thus, since $|\sin (2 \pi|Y| \sin A(Y))|$ $\leqq 2 \pi|Y| \sin A(Y)$, and since $t / g(t)$ is nondecreasing, it follows that the supremum is less than or equal to $\left[2 \sin A(Y) g\left((4 \sin A(Y))^{-1}\right)\right]^{-1}$, and this immediately implies the desired result.

Proof of Theorem 1. To begin with,

$$
F(r, \theta)=\int_{C} \exp (2 \pi i(r \theta, X)) d V_{X}
$$

where $d V_{X}=d x_{1} d x_{2}$.

By the divergence theorem, this last integral is equal, for $r \neq 0$, to

$$
(2 \pi i r)^{-1} \int_{\partial C} \exp (2 \pi i(r \theta, X))(\theta, n(X)) d S_{X},
$$

where $n(X)$ is the outward normal to $\partial C$ at the point $X$. Now the function $(\theta, n(X))$ is certainly $C^{1}$ in a neighborhood of $\partial C$, and is bounded on $\partial C$, together with its first derivatives, uniformly in $\theta$. The result thus follows from Lemma 1 , if we subdivide $\partial C$ into sufficiently small arcs.

Proof of Theorem 2. This follows from Lemma 2 in exactly the same way that Theorem 1 follows from Lemma 1 . (Take $g(t)=t^{\varepsilon}$ for part (1), and $g(t)=(\log t)^{1+\varepsilon}$ for part (2).)

2. In this section, we shall apply the results of $\S 1$ to some questions in the geometry of numbers. I should like to thank Professor Lars Hörmander for helpful conversations about the material in this section, and to thank Professor Atle Selberg for the initial suggestion which developed into Theorems 3, 4, and 5.

THEOREM 3. Suppose $C$ is a set of the type described in Theorem 1, and having area $A$. Suppose, moreover, that the interior of $C$ contains a point $p_{0}$, with respect to which $C$ is star-like, in the strong sense that no tangent line to $\partial C$ intersects $p_{0}$. For $\theta \in[0,2 \pi]$, let $L_{\theta}$ be the image of the integral lattice points under a counterclockwise rotation of size $\theta$. For $x>0$, let $N(x, \theta)$ be the number of points in $L_{\theta}$ which intersect 
the set $x C$, and define $R(x, \theta)$ to be the difference between $N(x, \theta)$ and the area of $x C$, i.e., $R(x, \theta)=N(x, \theta)-A x^{2}$. Then there exists a positive number $M$, such that

$$
\int_{0}^{2 \pi}|R(x, \theta)| d \theta \leqq M x^{2 / 3}
$$

REMARK. Considerably worse exponents can occur for specific values of $\theta$ (cf. [3] and [4]).

Proof. We shall make the temporary assumption that the point $p_{0}$ is the origin. Let $J(Y)$ be the indicator function of the set $C$. Then $J(Y / x)$ is the indicator function of the set $x C$, and the Fourier transform of $J(Y / x)$ is $x^{2} F(x Y)$, where $F(Y)$ is, as before, the Fourier transform of $J(Y)$.

Now

$$
N(x, \theta)=\sum_{N} J(\theta(N) / x)
$$

if we regard $\theta$ as a transformation in $S O(2)$.

Next let $\delta(Y)$ be a nonnegative $C^{\infty}$ function having support in the unit disk, and such that $\int_{R^{2}} \delta(Y) d V_{Y}=1$. Define $\delta_{\varepsilon}(Y)=\varepsilon^{-2} \delta(Y / \varepsilon)$. Then $\delta_{\varepsilon}(Y)$ has support in the disk $|Y| \leqq \varepsilon$, and has integral 1. Define

$$
J_{\varepsilon}(x, Y)=\int_{R^{2}} \delta_{\varepsilon}(Y-Z) J(Z / x) d V_{Z},
$$

and set $N_{\varepsilon}(x, \theta)=\sum J_{\varepsilon}(x, \theta(N))$. Now $J_{\varepsilon}(x, Y)$ is $C^{\infty}$ with compact support, so by the Poisson summation formula,

$$
\begin{aligned}
\sum J_{\varepsilon}(x, \theta(N)) & =\sum \delta_{\varepsilon}(\theta(N))\left[x^{2} F(x \theta(N))\right] \\
& =A x^{2}+\sum^{\prime} \delta_{\varepsilon}(\theta(N))\left[x^{2} F(x \theta(N))\right]
\end{aligned}
$$

where the prime on the summation sign means that the sum is taken over all nonzero integral lattice points.

Now we may clearly assume that the distance of $\partial C$ from the origin is initially as large as we please, and by taking this distance large enough, it follows from the star-like condition on $C$, that for small $\varepsilon>0, N_{\varepsilon}(x-\varepsilon, \theta) \leqq N(x, \theta) \leqq N_{\varepsilon}(x+\varepsilon, \theta)$. I.e., $N_{\varepsilon}(x-\varepsilon, \theta)-A x^{2} \leqq R(x, \theta) \leqq N_{\varepsilon}(x+\varepsilon, \theta)-A x^{2}$.

Now the last inequality implies that

$$
R(x, \theta) \leqq A\left[(x+\varepsilon)^{2}-x^{2}\right]+\sum^{\prime} \delta_{\varepsilon}(\theta(N))\left[(x+\varepsilon)^{2} F((x+\varepsilon) \theta(N))\right]
$$

But $\left|\delta_{\varepsilon}(Y)\right| \leqq M^{\prime}(1+\varepsilon|Y|)^{-1}$, for some $M^{\prime}>0$, so inserting the estimate for $F((x+\varepsilon) \theta(N))$ from Theorem 1 , we get

$$
R(x, \theta) \leqq 2 A x \varepsilon+A \varepsilon^{2}+M^{\prime}(x+\varepsilon)^{1 / 2} \sum^{\prime}(1+\varepsilon|N|)^{-1}|N|^{-3 / 2} \Phi(\theta(N /|N|)) .
$$


If, now, we define $\varepsilon=x^{-1 / 3}$, it follows that

$$
R(x, \theta) \leqq O\left(x^{2 / 3}\right)+M^{\prime}(x+\varepsilon)^{1 / 2} \sum^{\prime}(1+\varepsilon|N|)^{-1}|N|^{-3 / 2} \Phi(\theta(N /|N|)),
$$

with $O\left(x^{2 / 3}\right)$ independent of $\theta$.

By a similar process, we can show that

$$
R(x, \theta) \geqq-\left\{O\left(x^{2 / 3}\right)+M^{\prime}(x-\varepsilon)^{1 / 2} \sum^{\prime}(1+\varepsilon|N|)^{-1}|N|^{-3 / 2} \Phi(\theta(N /|N|))\right\},
$$

and this implies that

$$
|R(x, \theta)| \leqq M^{\prime \prime}\left\{x^{2 / 3}+x^{1 / 2} \sum^{\prime}(1+\varepsilon|N|)^{-1}|N|^{-3 / 2} \Phi(\theta(N /|N|))\right\}
$$

for some $M^{\prime \prime}>0$. I.e.,

$$
\int_{0}^{2 \pi}|R(x, \theta)| d \theta \leqq M^{\prime \prime}\left\{x^{2 / 3}+x^{1 / 2} \sum^{\prime}(1+\varepsilon|N|)^{-1}|N|^{-3 / 2} \int_{0}^{2 \pi} \Phi(\theta(N /|N|)) d \theta\right\}
$$

Now $\int_{0}^{2 \pi} \Phi(\theta(N /|N|)) d \theta$ is independent of $N$, so

$$
\int_{0}^{2 \pi}|R(x, \theta)| d \theta \leqq M^{\prime \prime}\left\{x^{2 / 3}+x^{1 / 2} \sum^{\prime}(1+\varepsilon|N|)^{-1}|N|^{-3 / 2}\right\}
$$

for some $M^{m}>0$. Furthermore,

$$
\Sigma^{\prime}=\sum_{|N|<1 / \varepsilon}^{\prime}+\sum_{|N| \geq 1 / \varepsilon}
$$

and if we estimate the last two sums by comparing them with integrals, recalling that $\varepsilon=x^{-1 / 3}$, we find that the estimates are identical, and that the joint estimate is $O\left(x^{1 / 6}\right)$. I.e.,

$$
x^{1 / 2} \sum^{\prime}(1+\varepsilon|N|)^{-1}|N|^{-3 / 2}=O\left(x^{2 / 3}\right)
$$

so

$$
\int_{0}^{2 \pi}|R(x, \theta)| d \theta \leqq M x^{2 / 3}, \text { for some } M>0 .
$$

To complete the proof, note that even if $C$ does not satisfy our temporary hypothesis about the origin, there is certainly some translate $C^{*}=t(C)$ which does. Moreover, the translation $t$ determines, in an obvious way, a family $t_{x}(x>0)$ of translations having the property that $t_{x}(x C)=x C^{*}$. If, now, we define $J^{*}(Y)$ to be the indicator function of the set $C^{*}$, and define $J_{\varepsilon}^{*}(x, Y)$ to be the convolution of $J^{*}(Y / x)$ with $\delta_{\varepsilon}(Y)$, then it follows, since $N(x, \theta)=\sum J^{*}\left(\left[t_{x} \theta(N)\right] / x\right)$, that

$$
\sum J_{\varepsilon}^{*}\left(x-\varepsilon, t_{x} \theta(N)\right) \leqq N(x, \theta) \leqq \sum J_{\varepsilon}^{*}\left(x+\varepsilon, t_{x} \theta(N)\right), \text { for small } \varepsilon>0 .
$$

If, finally, we note that for fixed $x, y$, and $\theta$, the Fourier transform of $J *\left(\left[\mathrm{t}_{x} \theta(Y)\right] / y\right)$ differs from that of $J^{*}([\theta(Y)] / y)$ by only an exponential factor of the form 
$\exp (2 \pi i(W, Y))$, where $W$ is a real vector, it becomes clear that we can apply our previous arguments, since the relevant estimate for the absolute value of the Fourier transform will not be changed.

The following theorem is a consequence of (2) of Theorem 2 , in very much the same way that Theorem 3 was a consequence of Theorem 1. We shall omit the proof, since it is so similar to the proof of Theorem 3.

Theorem 4. Suppose $C$ is a polygon. Define $R(x, \theta)$ as in Theorem 3. Then for every $\varepsilon>0$, there exists a number $M(\varepsilon)>0$, such that

$$
\int_{0}^{2 \pi}|R(x, \theta)| d \theta \leqq M(\varepsilon)(\log x)^{3+\varepsilon} .
$$

The following theorem is similar to a theorem of Kendall [2], with the important difference that we are admitting motions other than pure translations. This can be essential, if $\partial C$ has points of zero curvature.

THEOREM 5. Suppose $G$ is the group of all rigid motions of the plane having the form $\theta t$, where $\theta \in S O(2)$, and $t$ is a translation. Let I be the subgroup of integral translations, and define $H$ to be the quotient space G/I. Then $H$ is topologically the product of $S O(2)$ wth the torus $T^{2}$, and $H$ has a unique normalized invariant measure dh, which is, in fact, precisely the product measure. We can clearly regard $H$ as operating on the integral lattice-points in a well-defined way, and for $h \in H$, we define $L_{h}$ to be the image of the integral lattice-points under $h$. Suppose $C$ is a set of the type described in Theorem 1. For $h \in H$, define $R(x, h)$ to be the difference $N(x, h)$ $-A x^{2}$, where $A$ is the area of $C$, and $N(x, h)$ is the number of points in $L_{h}$ which intersect $x C$. Then there exists $M>0$, such that $\int_{H}(R(x, h))^{2} d h \leqq M x$.

Proof. If we fix $\theta, R(x, h)$ becomes a function on $T^{2}$, and it is easy to see that its Fourier series is

$$
\sum^{\prime} x^{2} F(x \theta(N)) \exp (2 \pi i(N, t)), \quad\left(t \in T^{2}\right),
$$

where $F(Y)$ is, as before, the Fourier transform of the indicator function of the set $C$.

Now

$$
\begin{aligned}
\int_{H}(R(x, h))^{2} d h & =\int_{S O(2)} d \theta \int_{T^{2}}(R(x, h))^{2} d t \\
& =\int_{S O(2)}\left(\Sigma^{\prime}\left|x^{2} F(x \theta(N))\right|^{2}\right) d \theta
\end{aligned}
$$

by Parseval's equality. Using the estimate from Theorem 1, and, in particular, the fact that $\Phi(\theta)$ is $L^{2}$, the desired result follows immediately.

REMARKS. 1. It would, I think, be interesting to investigate the analogue of Theorem 5 , for the case in which $H=S L(2, R) / S L(2, Z)$, and the summation is taken over the primitive integral lattice-points. 
2. It is possible, in some cases, to use the estimate for $\Phi(\theta)$ near a singularity to estimate the quantity $R(x, \theta)$ of Theorem 3 for specific values of $\theta$. One can also show that an estimate of the form $O\left(x^{2 / 3}\right)$ is valid for almost all $\theta \in[0,2 \pi]$.

\section{REFERENCES}

1. E. Hlawka, Ubber Integrale auf Konvexen Körpern. I, Monatsh. Math. 54 (1950), 1-36.

2. D. G. Kendall, On the number of lattice-points inside a random oval, Quart. J. Math. Oxford Ser. 19 (1948), 1-26.

3. B. Randol, A lattice-point problem, Trans. Amer. Math. Soc. 121 (1966), 257-268.

4. J. G. van der Corput, Over roosterpunten in het platte vlak, Noordhoff, Groningen, 1919.

YALE UNIVERSITY,

New Haven, Connecticut 